\title{
Early Adaptation of Small Intestine After Massive Small Bowel Resection in Rats
}

\author{
Jie Chen ${ }^{1}$; Zhen Qin ${ }^{1}$; Hongmei Shan ${ }^{2}$; Yongtao Xiao ${ }^{2}$; Wei Cai ${ }^{1,2,}$ \\ ${ }^{1}$ Department of Pediatric Surgery, Xin Hua Hospital, Shanghai Jiao Tong University, Shanghai, China \\ ${ }^{2}$ Shanghai Key Laboratory of Pediatric Gastroenterology and Nutrition, Shanghai Institute of Pediatric Research, Shanghai Jiao Tong University, Shanghai, China \\ *Corresponding author: Wei Cai, Shanghai Key Laboratory of Pediatric Gastroenterology and Nutrition, Shanghai Institute of Pediatric Research, Shanghai Jiao Tong University, \\ Shanghai, China. Tel:+86-2125078425, E-mail: caiw1978@163.com
}

Received: July 30, 2014; Accepted: December 22, 2014

\begin{abstract}
Background: It is important that the residual bowel adapts after massive resection. The necessary intestinal adaptation is a progressive recovery from intestinal failure through increase in absorptive surface area and functional capacity and includes both morphological and functional adaptations.

Objectives: The aim of this study was to investigate intestinal morphological and functional adaptations of small bowel syndrome (SBS) model rats (SBS1W) 7 days after bowel resection.

Materials and Methods: Male sprague-dawley rats ( $n=20$ /group) underwent either a 75\% proximal small bowel resection (SBSIW group) or a control operation (control group). Markers of morphological adaptation were revealed by TEM analysis of H\&E-stained tissue samples. The intestinal barrier condition was assessed by BT, and sIgA concentration in intestinal mucus was measured by ELISA. Contractility and the slow wave rhythm of the entire intestinal remnant were measured and recorded.

Results: The SBS1W group experienced more weight loss than control group and had a clearly different intestinal morphology as revealed in TEM images. Compared with control rats, the SBSIW group had a lower sIgA concentration in intestinal mucus and higher BT to lymph nodes (70\%vs 40\%; level I), portal blood (40\% vs 10\%; level II), and peripheral blood (60\% vs 30\%; level III). Disorder of spontaneous rhythmic contraction, irregular amplitude, and slow frequency were detected in the SBSIW group by a muscle strips test. Similarly, the slow wave of the entire intestinal remnant in the SBSIW group was irregular and uncoordinated.

Conclusions: The finding of intestinal adaptation following massive SBR in SBSIW rats provides more understanding of the mechanisms of progressive recovery from the intestinal failure that underlies SBS. The mechanical, chemical, immunological, and biological barriers were all impaired at 7 days following bowel resection, indicating that the SBS model rats were still in the intestinal adaptation phase.
\end{abstract}

Keywords: Short Bowel Syndrome; Compensation; Bacterial Translocation

\section{Background}

Short bowel syndrome(SBS) is described as an intestinal failure following massive resection of small bowel that results in malnutrition and a malabsorptive state (1). The amount of remaining bowel generally correlates with the degree of malabsorption and, consequently, the need for parenteral nutrition (PN) or specialized enteral nutrition (2). It is important for the residual bowel to adapt following massive resection by progressively recovering from intestinal failure with increase of absorptive surface area and functional capacity (3).

Several factors correlated with intestinal adaptation following small bowel resection (SBR) have been reported in the literature. Small intestinal bacterial overgrowth (SIBO) due to alterations in anatomy, motility, and secretion, is known to be an independent negative factor impeding adaptation of the small intestine after massive bowel resection in children $(4,5)$. It is also known that the barrier function of the intestine following SBR is usually impaired, resulting in bacterial trans- location (BT) (6). Reduction of gut-associated lymphoid tissue, following the SBR-related reduction of bowel length and intestinal transit time, leads to prolonged contact with luminal bacteria and may impair the local immune response to intestinal bacteria.

In contrast to what is known regarding SBR-induced functional changes and intestinal adaptations in mucosal and ISM layers, the contractile function of ISM and its contribution to the adaptive response remain largely unknown. Moreover, there is inconsistency in the literature about whether there is an adaptive change in intestinal peristalsis in the early phase after SBR.

\section{Objectives}

In our study, we focused on SBR-induced changes in morphology, proliferation of both the mucosal and ISM layers, and contractility of both ISM and the all-layer intestine samples from SBS model rats (SBS1W) in the early phase of intestinal adaptation following SBR. 


\section{Materials and Methods}

\subsection{Experimental Design and Animal Model}

Forty male sprague-dawley rats weighing 250 300 g were obtained from the experimental animal center of Shanghai Jiao Tong University School of Medicine. The rats were divided randomly into two experimental groups ( $n=20$, each). Group A rats (control) underwent bowel transection and anastomosis. Group B rats (SBS1W) underwent 75\% SBR (7). Animals were kept fasting for 16 hours prior to laparotomy and intestinal surgery, which were performed as previously described (8).

All experimental protocols were approved by the local Animal Care Committee and conformed to the Guide for the Care and Use of Laboratory Animals published by the Science and Technology Commission of the People's Republic of China (STCC Publication No. 2, revised 1988).

\subsection{Bacterial Translocation}

Investigation of BT of lactobacilli was performed separately from bacterial cultures of enteric origin using special agars (XLD/Hektone, McConkey, blood and phenyl agar, anaerobic blood agar + amikacin, Rogosa agar, and Saburaud agar). Bacterial translocation was considered positive if a bacterial culture of enteric origin was detected in at least one specimen. Bacterial translocation was stratified into three levels: (a) local, level I (mesenteric lymph nodes), (b) regional, level II (portal blood), and (c) systemic, level III (peripheral blood).

\subsection{Histological Examination}

At the time of harvest, a 30-mm segment of mid-ileum was removed and opened along the mesenteric border (9). Tissues were fixed in $4 \%$ paraformaldehyde and embedded in paraffin. Sections $(5 \mu \mathrm{m})$ were stained with hematoxylin and eosin ( $\mathrm{H} \& \mathrm{E}$ ) and examined using light microscopy $(10,11)$.

\subsection{Transmission Electron Microscopy}

Small intestinal tissue specimens and smooth muscle samples were fixed in a standard protocol (12) and examined with a Leitz Diaplan microscope using phase contrast microscopy. Ultra-thin sections of the specimens were stained with uranyl acetate (10 minutes) and counterstained with lead citrate ( 5 minutes) before being viewed with a Philips CM10 transmission electron microscope (13). Microvilli height evaluations were made according to a standard randomized protocol in 10 areas in the middle region of the villi. Micrographs containing approximately 24 microvilli were obtained and printed at the final magnification of $17500 \times$. A blinded, histological assessment was made of each micrograph.

\subsection{Detection of Secretory Immunoglobulin A (sIgA) in Intestinal Mucus}

Mucus samples were obtained using a modification of a technique described by Elson et al. (14). Briefly, the small intestine removed from each animal was rinsed in $10 \mathrm{~mL}$ of $\mathrm{pH} 7.4$ phosphate buffered saline (PBS) to collect intestinal mucus content. Then, the intestinal content was vigorously vortexed and centrifuged for 30 minutes at 300 $\mathrm{g}$ at $4^{\circ} \mathrm{C}$. The supernatant was transferred to a new tube and levels of sIgA in intestinal mucus samples were determined by ELISA.

\subsection{Measurement of Muscle Contractile Activity}

At the time of harvest, the intestine was opened, exposing the mucosa and allowing muscle strips to be obtained. Each muscle strip was 15-20 mm long and 2-3 $\mathrm{mm}$ wide. Contractile activity of each muscle strip was recorded using the SMUP-E biological signal processing system (Chengdu Equipment Factory). Physiography was used to record spontaneous contraction, absolute force, and rhythm of normal and distended intestinal circular muscle strips $(15,16)$.

\subsection{Extracellular Recording Experiment}

An $80-\mathrm{mm}$ mid-segment of the remaining small bowel was removed, and then the bowel segment was bathed in an extracellular recording device. The SMUP-E biological signal processing system (Chengdu Equipment Factory) and MFLab 200 software (Shanghai Medical College, China) were used to record spontaneous contraction, absolute force and rhythm of the intestinal segments.

\subsection{Statistical Analysis}

The results are expressed as mean \pm SD. Data were analyzed using SPSS 17.0 (SPSS Inc., Chicago, IL). The results of assessing bacterial content in fecal samples were expressed in log10 copy numbers g1 wet weight. The differences of all the parameters among groups were first compared by Kruskal-Wallis ANOVA; when a significant difference among groups was found, then the differences between the two groups were determined using the Mann-Whitney U-test. A P value of $<0.05$ was considered significant.

\section{Results}

\subsection{Body Weight}

The two groups began with the same mean body weight, and the SBS1W group showed an early decrease followed by an increase in body weight over time (Figure 1). The SBS1W group had a greater weight loss than the control group.

\subsection{Bacterial Translocation}

The results of bacterial translocation are shown in Table 1 and Table 2. In each level of BT, bacterial translocation of the experimental group is significantly higher than that of control group. 
Chen Jet al.

\subsection{Morphological Changes in Intestinal Bowel}

Histological staining indicated that microvilli from the mucosal tissues of small intestine were longer and disrupted in SBS1W rats compared with those from intact mucosa samples from the control group (Figure 2). SBS1W rats showed a significant increase $(P<0.01)$ compared with controls in villus height and crypt depth (Table 3 ). In the SBS1W intestine, there was a marked increase in the thickness of both the longitudinal and circular muscle layers (Figure 3).

\subsection{Morphological Changes of the Hypertrophic Muscle Cells}

Transmission electron microscopy showed a difference in the tight junction of microvilli between the two study groups. Hypertrophic muscle cells in the SBS1W group showed myofilaments and organelles typical of smooth muscle cells. However, mitochondria and smooth sarcoplasmic reticulum appeared to be more prominent in muscle cells from the SBS1W group than those from the control group. Complex clusters of sarcoplasmic reticulum sacs were also observed near the cell surface in the SBS1W group (Figure 3). In addition, hypertrophic muscle cells from SBS1W rats showed large numbers of small sacs of sarcoplasmic reticulum throughout the cytoplasm than those from control rats. Gap junctions were consistently observed in the circular muscle of the control ileum. In the hypertrophic muscle (SBS1W group), cells nexuses were more numerous than in control muscles and were on processes that abutted neighboring muscle cells.

\section{5. sIgA Concentration in Intestinal Content}

The concentration of SIgA in the intestine was signifi- cantly less in the SBS1W group than that in the control group (Figure 4 ).

\subsection{Adaptive Changes of Smooth Muscle Function}

The function of smooth muscle in control group was characterized by large and relatively regular spontaneous monophasic contractions (Figure 5). However, 1 week after SBR surgery, the spontaneous activity was significantly altered and displayed an irregular pattern with lower amplitude and frequency $(\mathrm{P}<0.05$; Figure 5).

\subsection{Extracellular Recording}

The control group displayed typical ongoing rhythmic contractions, which are due to slow-wave-type electrical activity. In contrast, the SBS1W group showed dysrhythmia and irregularity due to uncoordinated spontaneous contractions and lack of slow-wave-type electrical activity (Figure 6). This result validates that disturbed gastrointestinal motility is due to disrupted slow-wave activity.

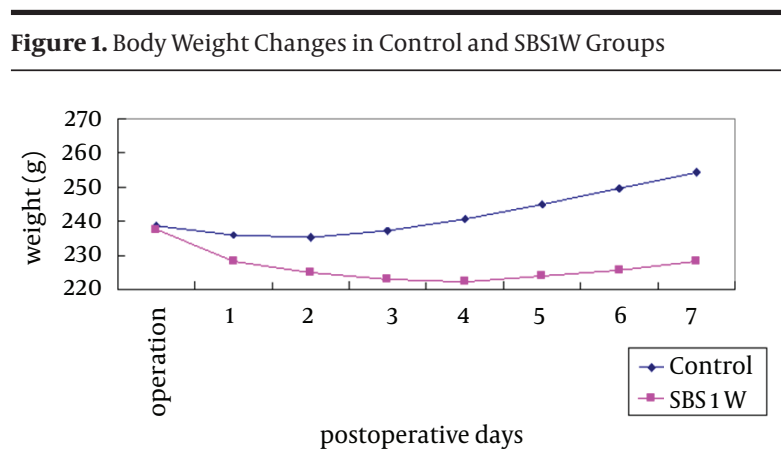

Rat body weight measured at different time points following either a 75\% small bowel resection (SBR1W) or transection (control) surgeries.

\begin{tabular}{|c|c|c|}
\hline Microorganism & SBS1W & Control \\
\hline \multicolumn{3}{|c|}{ Microorganisms of intestinal origin } \\
\hline Escherichia coli & $8(20)$ & $10(25)$ \\
\hline Enterococcus faecalis & $9(22.5)$ & $3(7.5)$ \\
\hline \multicolumn{3}{|l|}{ Contaminants } \\
\hline Stafilococco xylosus & $2(5)$ & $1(2.5)$ \\
\hline Staphylococcus saprophyticus & $1(2.5)$ & $1(2.5)$ \\
\hline
\end{tabular}

a Data are presented as No. (\%).

Table 2. Frequency of Bacteria Translocation to Blood and Peripheral Organs (\% of Number of Animals Exhibiting BT)

\begin{tabular}{lccc}
\hline Group & Level I Lymph Nodes & Level II Portal Blood & Level III Peripheral Blood \\
\hline SBS1W & $14(70)$ & $8(40)$ & $12(60)$ \\
Control & $8(40)$ & $2(10)$ & $6(30)$ \\
\hline
\end{tabular}



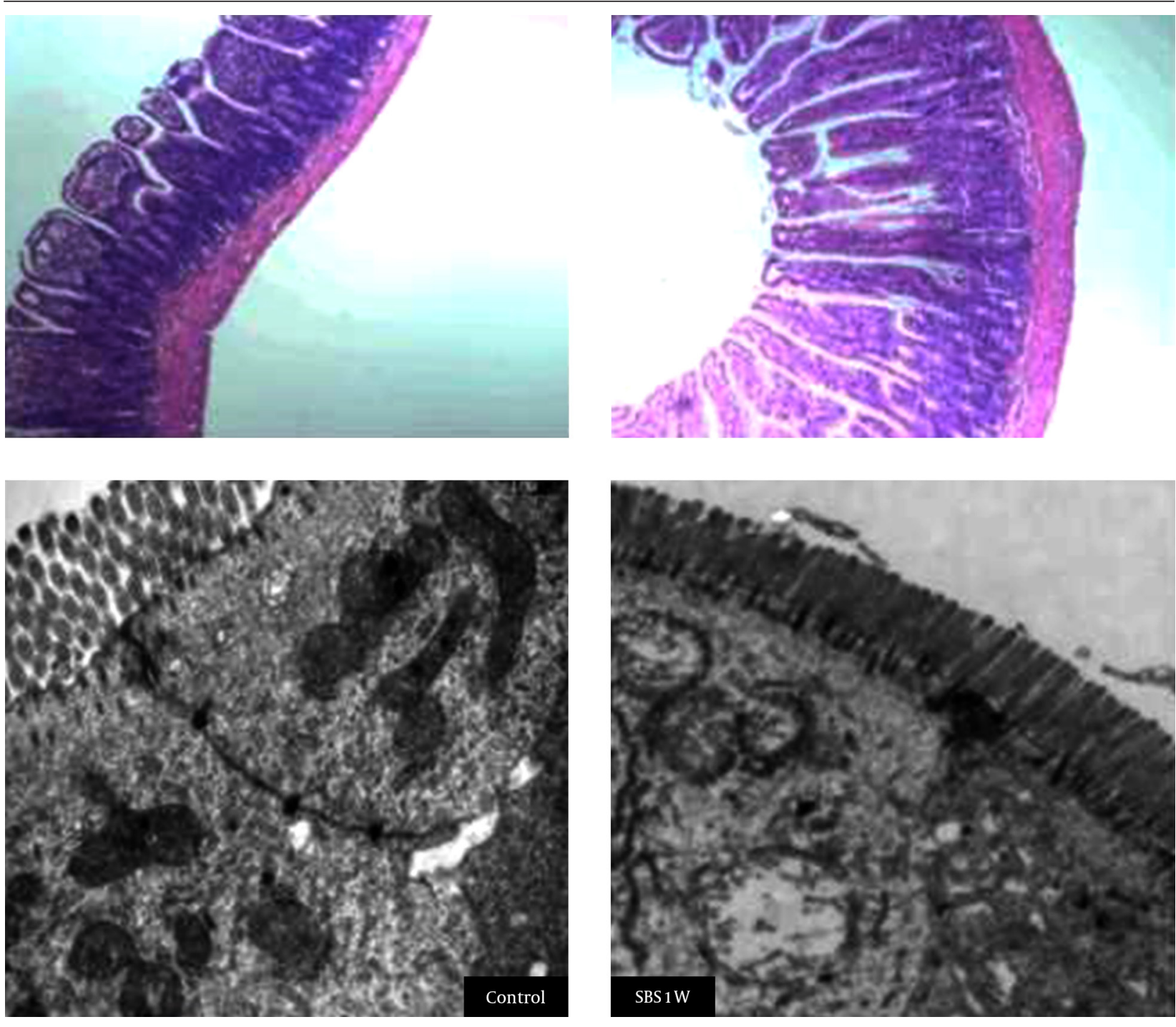

Cross-section of the ileum in control group and SBSIW group intestinal tissue stained with hematoxylin and eosin. At the ultrastructural morphological level, the height and density of mSBR-associated microvilli were significantly increased.

Table 3. Villus Height and Crypt Depth in Small Intestine Among Different Groups (mean \pm SD in $\mu \mathrm{m}$ )

\begin{tabular}{lcc}
\hline Group & SBS1W & Control \\
\hline Villus eight & $428 \pm 103^{\mathrm{a}}$ & $337 \pm 100^{\mathrm{a}}$ \\
Crypt depth & $233 \pm 60^{\mathrm{a}}$ & $204 \pm 48^{\mathrm{a}}$ \\
Villus eight $/$ crypt depth & $1.98 \pm 0.35$ & $1.73 \pm 0.63$ \\
\hline
\end{tabular}

$$
\text { a } \mathrm{P}<0.01 \text {. }
$$

\section{Discussion}

Intestinal adaptation includes both morphological and functional adaptations that occur in the residual intestine after SBR to restore the absorptive area and functional capacity (17). Structural or morphological adaptation in- cludes increasing bowel diameter and length, lengthening the villi, deepening the crypts, and increasing the rate of enterocyte proliferation. In addition, the contractile function of smooth muscle also plays a central role in the regu- 
lation of intestinal transit. In the current study, we confirmed that sstructural and functional adaptations involve both the mucosal and ISM layers in the rat SBSW1 model.

A hyperplastic response of the mucosa is prominent and results in elongated villi with increased cellularity and deeper crypts $(17,18)$. This villous hyperplasia is associated with a decrease in apoptosis, which leads to increases in RNA, DNA, and protein content $(19,20)$. In our study, SBS1W rats showed a significant increase (vs control) in villus height and crypt depth. Although the precise mechanisms of these adaptations are not clear, various agents (e.g. bombesin, transforming growth factor- $\beta$, growth hormone, melatonin, and epidermal growth factor) have been suggested to have both trophic effects on bowel mucosa and beneficial effects on intestinal adapta- tion (21). These results suggest that a possible initiating factor for intestinal adaptation is the insufficient and decompensated intestinal absorption area.

The integrated intercellular junction of intestinal mucosa keeps the intestinal microenvironment stable and guards against BT. We found that these important tight junctions were diminished and discontinuous in the SBS1W group (Figures 2 and 3). The disturbance in tight junctions resulted in changes in membrane permeability that was evidenced by increases in SIBO (Table 1) and BT (Table 2) following massive bowel resection in our study. SIBO due to alterations in anatomy, motility, and secretion is an independent negative factor that hinders adaptation of the small intestine in children after massive bowel resection (22).

Figure 3. Morphological Changes of the Intestinal Smooth Muscle and Ultra-Structure of the Intestinal Smooth Muscle Cells
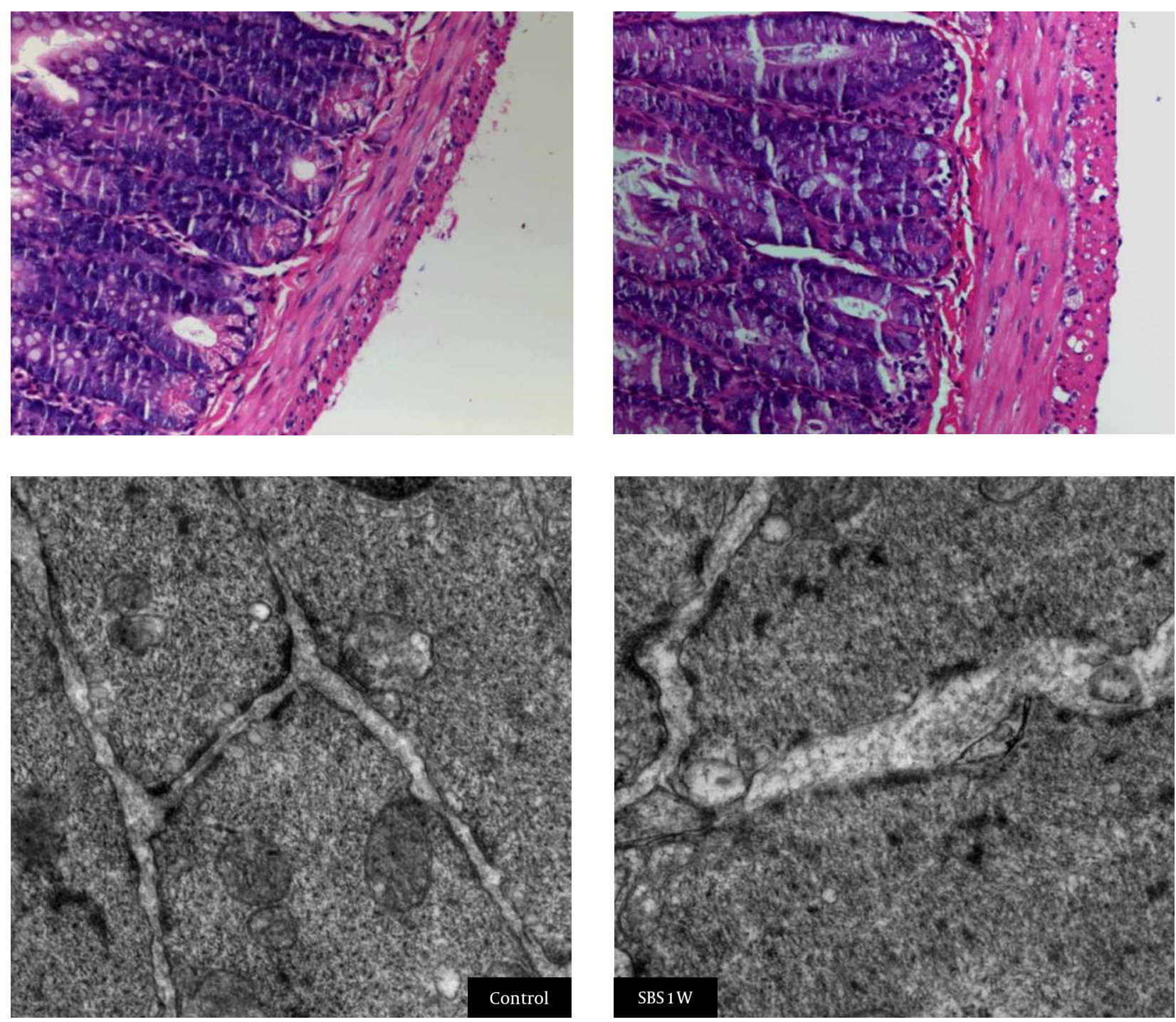

A marked increase is evident in the thickness of both the longitudinal and circular muscle layers in the SBSiW intestine sample (scale bar: $100 \mu \mathrm{m}$ ). At the ultrastructural morphological level, muscle cells in advanced states of hypertrophy showed the myofilaments and the organelles typical of smooth muscle cells. Mitochondria and smooth sarcoplasmic reticulum appeared to be more prominent in SBSIW samples. 
Figure 4. sIgA Levels in Intestinal Mucus in SBS1W and Control Groups

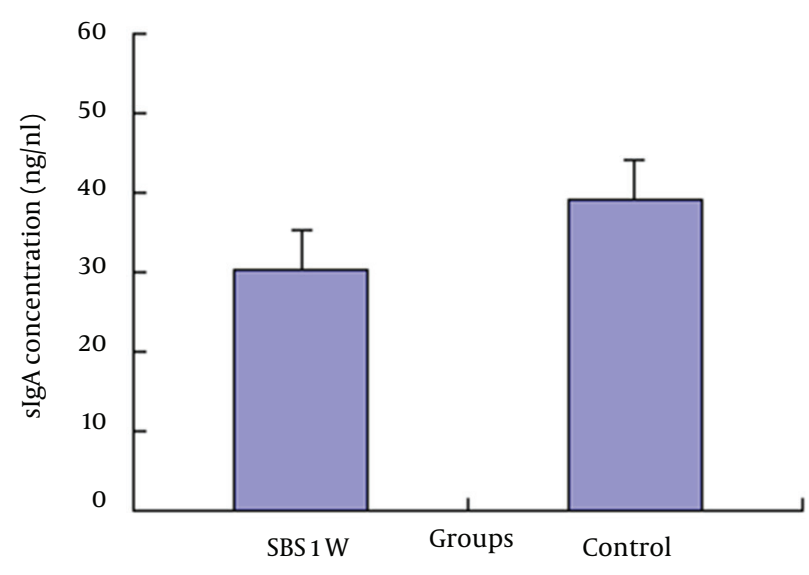

sIgA levels are expressed as ng/mL, $\left({ }^{*} \mathrm{P}<0.05\right)$.
In our study, massive small bowel resection resulted in 70\% BT to lymph nodes (level I), 40\% BT to portal blood (level II), and 60\% BT to peripheral blood (level III). There was significant difference in the concentration of SIgA in the intestine between the SBS1W group and the control group, which confirms that intestinal immunological barrier function after bowel resection was impaired.

The intestinal barrier is the interface between the luminal contents of the gut and the intestinal mucosa that harbors the gut-associated immune system.

We suggest that these mucosal occurrences are related to alterations in the intestinal anatomical and immunological microenvironment. In the current study, we also confirmed that resection-induced intestinal adaptation involves the ISM layer in SBS1W rats. The SBS1W rats exhibited a significant increase in the thickness of the smooth muscle layers in the remaining small bowel compared to controls (Figure 3). Hypertrophic muscle cells from the SBS1W group showed myofilaments and

Figure 5. Change in Spontaneous Rhythmic Contractions of the Intestinal Circular Muscle
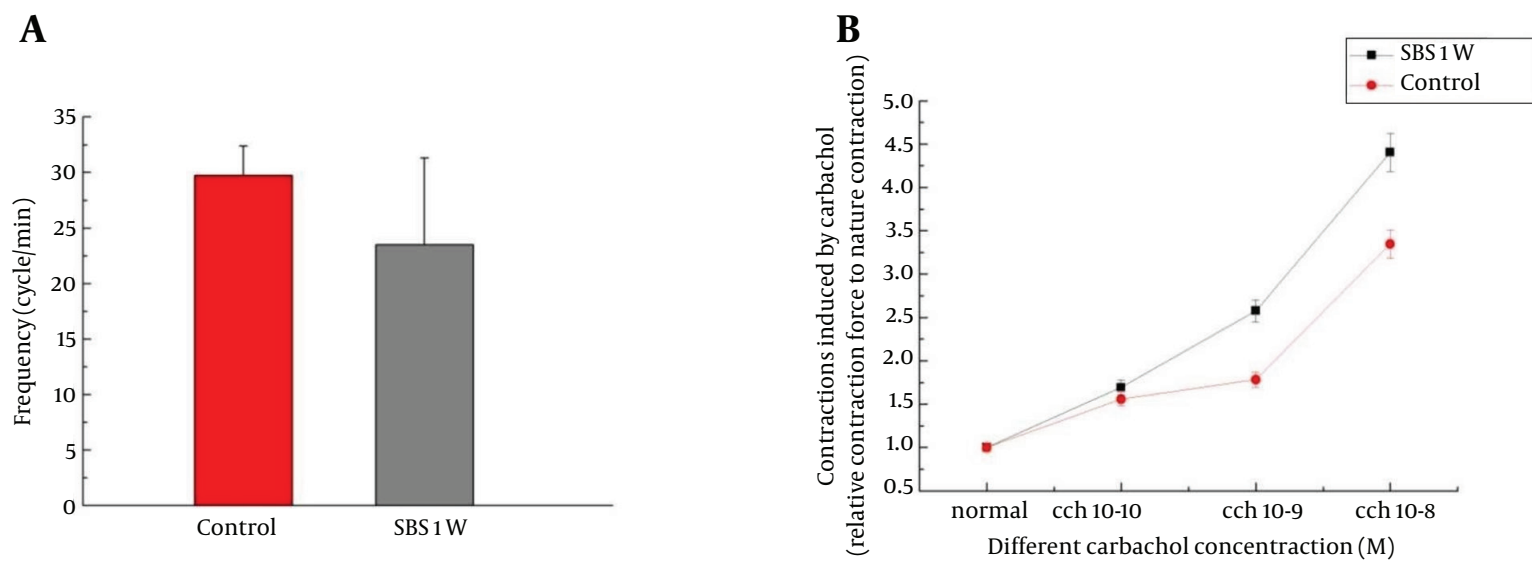

C $\operatorname{cch} 10^{-8}$

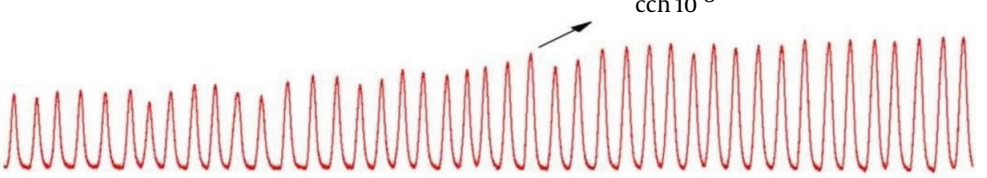

Control

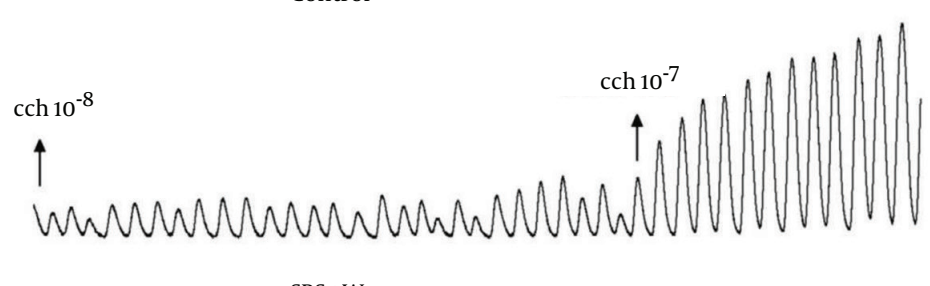

SBS $1 \mathrm{~W}$

The data illustrate typical traces of spontaneous contractions from different groups. Analytical data showing the frequency of spontaneous rhythmic contractions (A), contractions induced by different concentrations of carbachol in control and the SBS1W rat groups (B), and concentration-response curves for contraction of ileum strips in control and SBS1W rat groups in the presence of various concentrations of carbachol $(\mathrm{C}),\left({ }^{*} \mathrm{P}<0.05\right)$. 


\section{cunnumuntwhwhth}

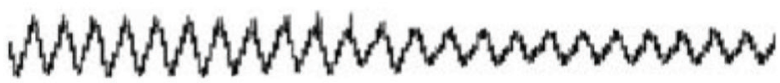

Control
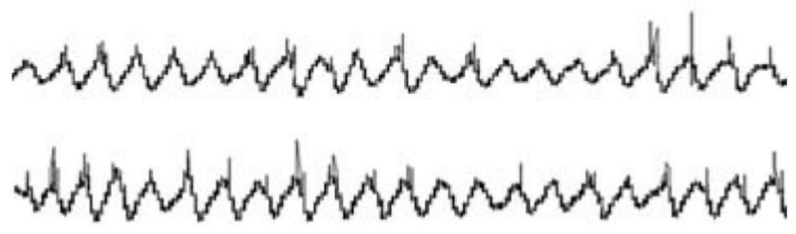

SBS $1 \mathrm{~W}$

The control group displayed typical ongoing rhythmic contractions, whereas the SBSIW group showed dysrhythmia and irregularity.

organelles that were typical of smooth muscle cells. However, mitochondria and smooth sarcoplasmic reticulum appeared to be more prominent in the SBS1W group than in the control group muscle cells. In addition, hypertrophic muscle cells (SBS1W group) showed large numbers of small sacs of sarcoplasmic reticulum scattered throughout the cytoplasm compared with the control group. Muscle cell nexuses were more numerous in SBS1W than in control muscles and were observed on processes abutting on neighboring muscle cells.

Our results regarding the function of smooth muscle and the entirety of the intestinal remnant are in agreement with reports indicating that adaptation is associated with the hypertrophy of smooth muscle, which is a physiological response to the increased functional requirement placed on the residual small bowel following resection $(23,24)$. Furthermore, hypertrophy of smooth muscle tissue produces distinct motility disorders in the intestinal remnant, resulting in malabsorption and loss of nutrients by diarrhea.

Another aspect of the involvement of smooth muscle in intestinal adaptation following SBR involves contractility. We found that the frequency of contractility significantly decreased at the 7th day, and spontaneous activity displayed a significantly altered irregular pattern with low amplitude and a lower frequency in SBS1W rats compared to control animals. Also, a reduction of rhythmic contractions and disruption of electrical slow waves determined with intracellular and extracellular recordings of smooth muscle in isolated segments of the remnant ileum tissue samples was shown to be associated with hypertrophy of smooth muscle tissue. Finally, the activity of the entire segment was uncoordinated and decompensated in our study (Figure 6).

The mechanical, chemical, immunological, and biological barriers after bowel resection were all impaired. It is feasible to speculate that these factors may have contributed to weight loss (Figure 1).

In summary, the present study shows that the SBS1W rat model of SBS displayed a decompensated phase that included changes in body weight, SIBO, BT, mucosal villus height and crypt depth, muscle cell morphology, sIgA content, and smooth muscle contractility. The process of intestinal adaptation was shown to have begun in the residual intestine at the 7th day after SBR, but it was far from the complete process of progressive recovery from intestinal failure at this early time.

\section{Acknowledgements}

This research was supported by the program for medengineering co-operation of Shanghai Jiao Tong University (Grant No.AE604102) and Natural Science Foundation of Shanghai, China (Grant No.14ZR1427200).

\section{Authors' Contributions}

Jie Chen and Wei Cai designed research; Jie Chen and Hongmei Shan performed research; Yongtao Xiao analyzed data; and Jie Chen and Zhen Qin wrote the paper.

\section{References}

1. Misiakos EP, Macheras A, Kapetanakis T, Liakakos T. Short bowel syndrome: current medical and surgical trends. J Clin Gastroenterol. 2007;41(1):5-18.

2. Duro D, Kamin D, Duggan C. Overview of pediatric short bowel syndrome. J Pediatr Gastroenterol Nutr. 2008;47 Suppl 1:S33-6.

3. DiBaise JK, Young RJ, Vanderhoof JA. Intestinal rehabilitation and the short bowel syndrome: part 1. Am J Gastroenterol. 2004;99(7):1386-95.

4. Sachdev AH, Pimentel M. Gastrointestinal bacterial overgrowth: pathogenesis and clinical significance. Ther Adv Chronic Dis. 2013;4(5):223-31.

5. Cole CR, Ziegler TR. Small bowel bacterial overgrowth: a negative factor in gut adaptation in pediatric SBS. Curr Gastroenterol Rep. 2007;9(6):456-62.

6. Quigley EM, Quera R. Small intestinal bacterial overgrowth: roles of antibiotics, prebiotics, and probiotics. Gastroenterology. 2006;130(2 Suppl 1):S78-90.

7. Chen J, Wen J, Cai W. Smooth muscle adaptation and recovery of contractility after massive small bowel resection in rats. Exp Biol Med (Maywood). 2012;237(5):578-84

8. Washizawa N, Gu LH, Gu L, Openo KP, Jones DP, Ziegler TR. Comparative Effects of Glucagon-Like Peptide-2 (GLP-2), Growth Hormone (GH), and Keratinocyte Growth Factor (KGF) on Markers of Gut Adaptation After Massive Small Bowel Resection in Rats.JPEN JParenter Enteral Nutr. 2004;28(6):399-409.

9. Chen I, Chen H, Sanders KM, Perrino BA. Regulation of SRF CArG-dependent gene transcription during chronic partial obstruction of murine small intestine. Neurogastroenterol Motil. 2008;20(7):829-42.

10. Storkholm JH, Zhao J, Villadsen GE, Hager H, Jensen SL, Gregersen $\mathrm{H}$. Biomechanical remodeling of the chronically obstructed Guinea pig small intestine. Dig Dis Sci. 2007;52(2):336-46.

11. Won KJ, Suzuki T, Hori M, Ozaki H. Motility disorder in experimentally obstructed intestine: relationship between muscularis inflammation and disruption of the ICC network. Neurogastroenterol Motil. 2006;18(1):53-61. 
12. Guo X, Huang X, Wu YS, Liu DH, Lu HL, Kim YC, et al. Down-regulation of hydrogen sulfide biosynthesis accompanies murine interstitial cells of Cajal dysfunction in partial ileal obstruction. PLoS One. 2012;7(11):e48249.

13. Chang IY, Glasgow NJ, Takayama I, Horiguchi K, Sanders KM, Ward SM. Loss of interstitial cells of Cajal and development of electrical dysfunction in murine small bowel obstruction. J Physiol. 2001;536(Pt 2):555-68.

14. Elson CO, Ealding W, Lefkowitz J. A lavage technique allowing repeated measurement of IgA antibody in mouse intestinal secretions. Journal of Immunological Methods. 1984;67(1):101-8.

15. Chen J, Du L, Xiao YT, Cai W. Disruption of interstitial cells of Cajal networks after massive small bowel resection. World J Gastroenterol. 2013;19(22):3415-22.

16. Qiu ZX, Mei B, Wu YS, Huang X, Wang ZY, Han YF, et al. Atrial natriuretic peptide signal pathway upregulated in stomach of streptozotocin-induced diabetic mice. World J Gastroenterol. 2010;16(1):48-55.

17. Alpers DH. How adaptable is the intestine in patients with shortbowel syndrome? Am J Clin Nutr. 2002;75(5):787-8.

18. Hanson WR, Osborne JW, Sharp JG. Compensation by the residual intestine after intestinal resection in the rat. II. Influence of post- operative time interval. Gastroenterology. 1977;72(4 Pt 1):701-5.

19. Tavakkolizadeh A, Whang EE. Understanding and augmenting human intestinal adaptation: a call for more clinical research. JPEN JParenter Enteral Nutr. 2002;26(4):251-5.

20. Qandeel HG, Alonso F, Hernandez DJ, Madhavan S, Duenes JA Zheng Y, et al. Peptide absorption after massive proximal small bowel resection: mechanisms of ileal adaptation. J Gastrointest Surg. 2011;15(9):1537-47.

21. Gill RK, Anbazhagan AN, Esmaili A, Kumar A, Nazir S, Malakooti J, et al. Epidermal growth factor upregulates serotonin transporter in human intestinal epithelial cells via transcriptional mechanisms. Am J Physiol Gastrointest Liver Physiol. 2011;300(4):G627-36.

22. Wessel JJ, Kocoshis SA. Nutritional management of infants with short bowel syndrome. Semin Perinatol. 2007;31(2):104-11.

23. Goulet O, Baglin-Gobet S, Talbotec C, Fourcade L, Colomb V, Sauvat $\mathrm{F}$, et al. Outcome and long-term growth after extensive small bowel resection in the neonatal period: a survey of 87 children. EurJ Pediatr Surg. 2005;15(2):95-101.

24. Martin CA, Bernabe KQ, Taylor JA, Nair R, Paul RJ, Guo J, et al. Resection-induced intestinal adaptation and the role of enteric smooth muscle. J Pediatr Surg. 2008;43(6):1011-7. 